Sport Humanities

\title{
Effects of different verbal commands on perceptual, affective, and physiological responses during running
}

\author{
Sandro dos Santos Ferreira ${ }^{1}$ (D), Armando Luiz Bomfim Neto $^{2}$ (D), Lucio Follador ${ }^{2}$ (D), \\ Erick Doner Santos de Abreu Garcia ${ }^{2}$ (D), Vinícius Ferreira dos Santos Andrade ${ }^{2}$ (D), \\ Sergio Gregório da Silva ${ }^{2}$ (iD \\ ${ }^{1}$ Instituto Federal do Espírito Santo, Piúma, ES, Brazil; ${ }^{2}$ Universidade Federal do Paraná, \\ Curitiba, PR, Brazil. \\ Associate Editor: Giselle Helena Tavares, Universidade Federal de Uberlândia, Uberlândia, MG, \\ Brazil.
}

\begin{abstract}
Aims: To compare the effects of different verbal commands on the perceptual, affective, and physiological responses during running. Methods: Twenty men who regularly performed aerobic exercise and running (mean age: $30.7 \pm 10.1$ years; mean $\mathrm{VO}_{2 \max }: 55.3 \pm 7.1 \mathrm{~mL} \cdot \mathrm{kg}^{-1} \cdot \mathrm{min}^{-1}$ ). All subjects completed four exercise sessions: a familiarization trail; three running sessions consisting of a self-selected pace defined by one of the verbal commands: low, moderate, or high. These conditions were counterbalanced. Each running session lasted $20 \mathrm{~min}$. Affective responses (measured by the Feeling Scale and the Felt Arousal Scale) and physiological (measured by the hate rate and treadmill speed) were evaluated during each exercise session. Session RPE (OMNI-RES) was measured 15 min after each exercise session. Results: The perceptual and physiological responses were different for all verbal commands, with the intensity increasing according to the verbal commands given during running $(p<0.01)$. Affective responses to low and moderate exercise were similar and more pleasant than at high intensities; however, the affective responses to all verbal commands were positive. Conclusion: The results of the present study suggest that verbal commands can be used in running prescriptions. A moderate verbal command can promote greater perceptual and physiological stimuli than a low verbal command, without significant changes in affective responses.
\end{abstract}

Keywords: affective responses, RPE, verbal command, physiological responses, running.

\section{Introduction}

Exertional mediators have been commonly used in the assessment, prescription, and monitoring of exercise practice and can be classified as physiological, psychosocial, and performance mediators ${ }^{1}$. Physiological mediators involve respiratory-metabolic (oxygen uptake), peripheral $(\mathrm{pH}$, acid lactic), and non-specific (skin and body core temperature) categories; psychosocial mediators involve affective (pleasure and displeasure sensation), cognitive (association/dissociation; self-efficacy), perceptual process (somatic perceptual), and social/situation (social situation) mediators; and performance-related mediators involve speed/pace and time/distance traveled ${ }^{1-4}$. These mediators may function individually or collectively during exercise.

Physiological and performance-related mediators show features related to the development of performance, while psychosocial mediators, demonstrated as psychological variables, can influence performance or how exercise intensity can affect psychological variables ${ }^{5-7}$. The integration of exertional mediators reports the perceptual responses, which can be defined as the ability to detect and interpret organic sensations while performing exercise ${ }^{2}$.

The rating of perceived exertion (RPE) is the principal perceptual method used to prescribe and monitor exercise $^{8}$. In the last decades, the RPE has evolved and has become a common method because of its lower cost and ease of use ${ }^{1,9}$. Moreover, psychosocial mediators as the affective response have been analyzed, along with the RPE and other physiological mediators, to understand the relationship between exercise intensity and exercise adherence $^{10,11}$.

Affective responses can be described as the pleasure and displeasure sensations experienced during exercise $^{10,12}$. Investigations of the aerobic exercise demonstrated that the transition from aerobic to anaerobic metabolism could influence the responses during exercise. Intensities above the ventilatory threshold can promote negative affective responses, in contrast to those near or below the ventilatory threshold ${ }^{13,14}$. Vandoni, Codrons ${ }^{15}$ demonstrated in their investigation that subjects participating in vigorous-intensity exercise reported experiencing less pleasure than those who undertook moderate- 
intensity exercise. In a recent study conducted by Timme and Brand ${ }^{16}$, the affective responses declined as the exercise intensity increased during the incremental test, and high inter-individual variability was reported.

In order to strike a balance and integrate the different exertion mediators, exercise at a self-selected intensity has been used as a strategy for exercise prescription ${ }^{17}$. Selfselected intensity is when the participant chooses the preferred intensity to perform the proposed exercise ${ }^{18}$. In both active and sedentary people, a self-selected intensity can provide a stimulus below or around the ventilatory threshold (VT), a condition sufficient to improve cardiorespiratory fitness and promote health benefits according to the recommendations for moderate and vigorous aerobic exercise intensities (64\%-95\% HRmax) $)^{19}$. Moreover, the self-selected intensity is associated with a positive affective response ${ }^{20,21}$. In an investigation by Freitas, Ferreira ${ }^{7}$ it was observed that obese women undertaking a 12-week exercise intervention reported more pleasurable affective responses when the exercise intensity was self-selected vs imposed. Dias, Simao ${ }^{22}$ found that recreational exercisers adjust the intensity in line with typical training recommendations ( $83.9 \%$ of peak heart rate).

Self-selected intensity can be a usual and practical method for prescribing exercise; however, there is a great intensity range around and below the VT where self-selected intensity can be realized without showing a significant negative affective response, and there is a wide inter-individual variation in this range ${ }^{17,18}$. To minimize the individual variability and explore the intensities that promote positive affective responses, in this study, we specified intensity ranges, based on verbal commands, so that the participant was directed to a specific intensity zone and thus was free to adjust their effort according to their characteristics within the prescribed intensity range.

In this study, we aimed to compare the effects of different verbal commands on the perceptual, affective, and physiological responses during running. The experimental hypothesis was that the participants would be able to adjust the intensity of exercise according to the proposed verbal commands, leading to differences in the perceptual and physiological responses under all conditions. However, the affective responses would remain positive under all conditions.

\section{Methods}

\section{Participants}

Twenty men who regularly performed aerobic exercise and running activities $(30.7 \pm 10.1 \mathrm{y})$ were enrolled. Subjects ran at least 3-5 times and covered 25-50 km every week. The inclusion criteria were as follows: (a) 1850 years of age; (b) negative responses to all questions in the Physical Activity Readiness Questionnaire (PAR-Q); (c) performance in a run test between 18 and $26 \mathrm{~min}$ $(5 \mathrm{~km})$; and (d) a personal statement of not having smoked in the last 12 months. The exclusion criteria were the presence of cardiovascular, metabolic, or orthopedic disease or any other contraindications as determined by medical history in the preceding 12 months. This study was approved by the Research Ethics Committee of the Federal Institute of Espirito Santo, Brazil, CAAE: 21856919.4.0000.5072. Following a comprehensive explanation of procedures, benefits, and risks, volunteers provided written informed consent. The sample size was based on anticipated medium-to-large effect size (Cohen's $\mathrm{f}=0.25-0.40$ ), a power level of 0.8 , an alpha criterion of 0.05 , and a non-sphericity correction of 0.75 . Power analysis indicated a sample size of 15-34 participants ( $G *$ Power 3.1).

\section{Experimental design}

All subjects completed four exercise sessions consisting of a familiarization trail and three running sessions at a self-selected pace defined by one of the verbal commands: low, moderate, or high. Each running session consisted of 20 min performed on different days, with at least $72 \mathrm{~h}$ between sessions, in a counterbalanced order. The Session Rating of Perceived Exertion (RPE-S), Feeling Scale (FS), Felt Arousal Scale (FAS), and Heart Rate (HR) were recorded during each experimental session. Participants were advised not to consume alcohol, caffeine, or practice vigorous physical activity $24 \mathrm{~h}$ before each test.

First Session: the participants answered a healthscreening questionnaire (PAR-Q); were familiarized with the RPE scale (OMNI-RES), FS, and FAS; and performed a $\mathrm{VO}_{2 \max }$ treadmill test. Anthropometric data for height and body mass were measured using a wall stadiometer $(0.1 \mathrm{~cm})$ and an electronic scale $(0.1 \mathrm{~kg})$.

For the $\mathrm{VO}_{2 \max }$ treadmill test, a modified protocol with a $1 \%$ inclination was used. The test started with $2 \mathrm{~min}$ at $9 \mathrm{~km} / \mathrm{h}$ and increased by $1 \mathrm{~km} / \mathrm{h}$ every $2 \mathrm{~min}$ up to $11 \mathrm{~km} \cdot \mathrm{h}^{-1}$. Then, the velocity increased by $0.5 \mathrm{~km} \cdot \mathrm{h}^{-1}$ every minute until maximal voluntary exhaustion. Before the test, subjects performed 3 min warm-up at $8 \mathrm{~km} \cdot \mathrm{h}^{-1}$. The subjects were encouraged verbally to continue the exercise to the point of exhaustion. The criteria required to achieve $\mathrm{VO}_{2 \max }$ were as follows: (a) a plateau of $\mathrm{VO}_{2}$ (changes $<150 \mathrm{~mL} / \mathrm{min}$ ), (b) respiratory exchange ratio $\geq 1.10$, and (c) heart rate (HR) within $10 \mathrm{bpm}$ of the maximum level expected for the subject's age16). HR (bpm) was measured every $5 \mathrm{~s}$ using a Polar monitoring system (Polar Electro ${ }^{\mathrm{TM}}$, Oy, Finland). A portable analyzer (Cosmed $\mathrm{K} 4 \mathrm{~b} 2$, Rome, Italy) was used to measure $\mathrm{O}_{2}$, carbon dioxide production $\left(\mathrm{CO}_{2}\right)$, and pulmonary ventilation $\left(\mathrm{V}_{\mathrm{E}}\right.$, STPD). The expired gases were collected and analyzed breath-by-breath.

The running session was self-selected by verbal commands. All subjects completed three exercise sessions 
defined by one of the verbal commands: low, moderate, and high. The information conveyed to the subjects was the following: "Based on your experience in training, running competitions, and your current fitness level, I want you to think what it would be like for you to run (continuously) at a (low/moderate/high) intensity." "Now, I would like you to self-select $20 \mathrm{~min}$ of running at a (low/ moderate/high) intensity." "It must be an intensity that seems appropriate to you." These conditions were counterbalanced. Each running session lasted $20 \mathrm{~min}$. Subjects could adjust the intensity after $1,5,10$, and 15 min during the exercise session. However, at no time, were subjects allowed to know their running speed. The subjects' physiological performance was measured during each exercise session $(5,10,15$, and $20 \mathrm{~min})$. Affective responses (assessed by the Feeling Scale and Felt Arousal Scale) were measured before; $5,10,15$, and $20 \mathrm{~min}$ into; and 5 and $10 \mathrm{~min}$ after the running session. RPE-S was measured $15 \mathrm{~min}$ after each exercise session.

\section{Measurement}

Affective responses.

The affective valence was determined using the scale of Hardy and Rejeski ${ }^{23}$. This instrument comprises an 11point scale, ranging from +5 ("very good") to -5 ("very bad"). The Felt Arousal Scale (FAS) was used to measure the perception of activation ${ }^{24}$. The scale comprises six levels of activation, ranging from low (1) to high (6). A high perception of activation can be characterized in the following ways: excitement, anxiety, or anger. Low activation appears as relaxation, boredom, or quiet. Affective responses were measured before; 5, 10, 15, and $20 \mathrm{~min}$ into; and 5 and $10 \mathrm{~min}$ after the running session, and they are shown by a circumplex model.

\section{Circumplex model}

This consists of an affective space divided into the following four quadrants: Quadrant 1 corresponds to a sense of high activation and displeasure (tension); quadrant 2 corresponds to a sense of high activation and pleasure (energy); quadrant 3 corresponds to a sense of low activation and displeasure (tiredness), and quadrant 4 corresponds to a sense of low activation and pleasure (calmness) $^{10}$.

\section{Rating of Perceived Exertion}

The Session Rating of Perceived Exertion (RPE-S) was determined using the RPE OMNI-RES scale ${ }^{25}$. This instrument consists of a 10-point Likert scale in which 0 indicates "extremely easy" and 10 indicates "extremely difficult." Participants were instructed on how to use the scale and perform docking procedures before the tests as described by Utter, Robertson ${ }^{25}$. The RPE-S was measured 15 min after each exercise session.
Physiological responses

During the exercise sessions, the HR was collected in the last $5 \mathrm{~s}$ of each minute. Average Treadmill speed $\left(\mathrm{km} \cdot \mathrm{h}^{-1}\right)$ was determined by the division of speeds observed at the 5, 10,15, and 20-min marks.

\section{Statistical analysis}

The analysis was performed using Statistical Package for the Social Sciences (SPSS) Statistics version 22.0 (IBM) for Windows. The Shapiro-Wilk test was used to analyze the data distribution. One-way analysis of variance (ANOVA) for repeated measures was used for comparisons between conditions, whereas two-way ANOVA was used for comparisons of condition $\times$ time. The main effects were analyzed using the Bonferroni post hoc test. When the assumptions of sphericity were violated, Greenhouse-Geisser corrections were employed. The magnitude of the effect was calculated by the partial eta squared $(\eta 2 p)$. Values of $0.01,0.06$, and 0.14 corresponded to small, medium, and large effect sizes, respectively. The magnitude of the effect under the conditions was expressed as Cohen's d effect sizes $(0.2,0.5$, and 0.8 for small, medium, and large effects, respectively). All the differences were considered significant at $\mathrm{p}<0.05$.

\section{Results}

Tables 1 and 2 show the anthropometric and physiological characteristics and the perceptual, affective, and physiological performance responses during exercise to verbal commands of low intensity (LI), moderate intensity (MI), and high intensity (HI).

\section{$R P E$}

There were significant differences between all verbal commands $\left(\mathrm{F}_{(2,38)}=160.149, \mathrm{p}=0.000, \mathrm{n}_{\mathrm{p}}^{2}=0.894\right)$. The RPE was significantly higher in MI $x$ LI $(\mathrm{p}<0.01$, $\mathrm{d}=2.546)$, HI $x$ MI $(\mathrm{p}<0.01, \mathrm{~d}=1.676)$, and HI $x \mathrm{LI}$ $(\mathrm{p}<0.01, \mathrm{~d}=4.293)$.

Table 1 - Anthropometric and physiological characteristics of the study participants.

\begin{tabular}{|c|c|}
\hline Variables & Mean \pm SD \\
\hline Age (years) & $30.7 \pm 10.1$ \\
\hline Body Mass (kg) & $74.7 \pm 11.7$ \\
\hline Height (cm) & $175.2 \pm 8.8$ \\
\hline BMI $\left(\mathrm{kg} \cdot \mathrm{m}^{2}\right)$ & $24.3 \pm 2.6$ \\
\hline $\mathrm{VO}_{2 \max }\left(\mathrm{mL} \cdot \mathrm{kg}^{-1} \cdot \mathrm{min}^{-1}\right)$ & $55.3 \pm 7.1$ \\
\hline $\mathrm{HR}_{\max }\left(\mathrm{b} \cdot \mathrm{min}^{-1}\right)$ & $189.3 \pm 8.5$ \\
\hline Treadmill speed $_{\max }\left(\mathrm{km} \cdot \mathrm{h}^{-1}\right)$ & $15.3 \pm 1.1$ \\
\hline
\end{tabular}


Table 2 - Perceptual, affective, and physiological performance responses during exercise.

\begin{tabular}{|c|c|c|c|}
\hline Variables & Low intensity & Moderate intensity & High intensity \\
\hline Treadmill speed $\left(\mathrm{km} \cdot \mathrm{h}^{-1}\right)$ & $8.8 \pm 0.8^{\mathrm{a} \&}$ & $10.5 \pm 1.1^{* \&}$ & $12.5 \pm 0.9^{\mathrm{a} \&}$ \\
\hline$\%$ Treadmill speed $\max _{\max }$ & $57.8 \pm 5.8^{* \mathrm{a}}$ & $68.8 \pm 7.1 * \&$ & $81.5 \pm 6.4^{\mathrm{a} \&}$ \\
\hline HR (bpm) & $144.7 \pm 10.6^{*^{\mathrm{a}}}$ & $157.1 \pm 9.1 * \&$ & $171.5 \pm 11.4^{\mathrm{a} \&}$ \\
\hline$\% \mathrm{HR}_{\max }$ & $76.5 \pm 6.0 *^{\mathrm{a}}$ & $83.1 \pm 6.1{ }^{* \&}$ & $90.6 \pm 5.8^{\mathrm{a} \&}$ \\
\hline RPE & $2.7 \pm 0.9^{* a}$ & $5.4 \pm 1.2 * \&$ & $7.5 \pm 1.3^{\mathrm{a} \&}$ \\
\hline Feeling Scale & $3.5 \pm 1.1^{\mathrm{a}}$ & $3.0 \pm 1.5^{\&}$ & $1.8 \pm 2.2^{\mathrm{a} \&}$ \\
\hline FAS & $4.1 \pm 1.2^{\mathrm{a}}$ & $4.1 \pm 1.2^{\&}$ & $4.7 \pm 1.2^{\&}$ \\
\hline
\end{tabular}

HR: Heart Rate; $\% \mathrm{HR}_{\max }$ : Percentage Maximal Heart Rate; FS: Feeling Scale; FAS: Felt Arousal Scale. $\left({ }^{*}\right)$ Difference between low and moderate; $\left({ }^{\mathrm{a}}\right)$ difference between low and high; $\left({ }^{\&}\right)$ difference between moderate and high $\mathrm{p}<0.05$.

\section{$H R$ and $\% H R$}

There were significant differences between all verbal commands. HR $\left(\mathrm{F}_{(2,38)}=59.586, \mathrm{p}=0.000, \mathrm{n}_{\mathrm{p}}^{2}=0.758\right)$ was significantly higher in MI x LI $(\mathrm{p}<0.01, \mathrm{~d}=1.255)$, HI x MI $(\mathrm{p}<0.01, \mathrm{~d}=1.396)$, and HI x LI $(\mathrm{p}<0.01$, $\mathrm{d}=2.435) . \% \mathrm{HR}_{\max }\left(\mathrm{F}_{(2,38)}=59.536, \mathrm{p}=0.000\right.$, $\left.\mathrm{n}_{\mathrm{p}}^{2}=0.759\right)$ was significantly higher in MI x LI $(\mathrm{p}<0.01$, $\mathrm{d}=1.091)$, HI x MI ( $<<0.01, \mathrm{~d}=1.26)$, and HI x LI $(\mathrm{p}<0.01, \mathrm{~d}=2.389)$. There were significant effects of time $\left(\mathrm{F}_{(1,352,25,686)}=82.787, \mathrm{p}<0.001, \mathrm{n}^{2}{ }_{\mathrm{p}}=0.813\right)$ and condition $\left(\mathrm{F}_{(2,38)}=59.247, \mathrm{p}<0.001, \mathrm{n}^{2} \mathrm{p}=0.757\right)$. In addition, there was a significant interaction between time and condition $\left(\mathrm{F}_{(2,678,50,879)}=5.745, \mathrm{p}<0.001\right.$, $\left.\mathrm{n}_{\mathrm{p}}^{2}=0.232\right)$. This finding demonstrates that the HR was always different for different conditions $(\mathrm{p}<0.01)$ (Figure 1). The effect of time occurred under all conditions; the result was higher at 10, 15, and 20 min than that at $5 \min (\mathrm{p}<0.01)$.

\section{Treadmill speed and \% Treadmill speed max}

There was a significant difference between all verbal commands. Treadmill speed $\left(\mathrm{F}_{(2,38)}=145.746, \mathrm{p}=0.000\right.$, $\left.\mathrm{n}_{\mathrm{p}}^{2}=0.885\right)$ was significantly higher in MI x LI $(\mathrm{p}<0.01$, $\mathrm{d}=1.768)$, HI x MI $(\mathrm{p}<0.01, \mathrm{~d}=1.99)$, and HI x LI

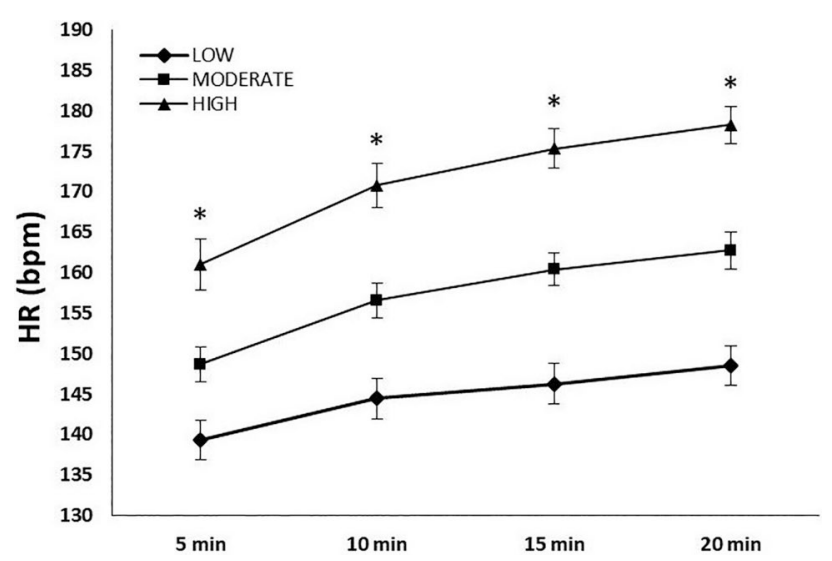

Figure 1 - HR (Heart Rate) during low, moderate, and high verbal commands. * Significant difference under all conditions. $(\mathrm{p}<0.01, \mathrm{~d}=4.345) . \%$ Treadmill $\operatorname{speed}_{\max }\left(\mathrm{F}_{(2,38)}=\right.$ 145.503, $\left.\mathrm{p}=0.000, \mathrm{n}_{\mathrm{p}}^{2}=0.882\right)$ was significantly higher in MI x LI $(\mathrm{p}<0.01, \mathrm{~d}=1.697)$, HI x MI $(\mathrm{p}<0.01$, $\mathrm{d}=1.879)$, and HI $x$ LI $(\mathrm{p}<0.01, \mathrm{~d}=2.243)$. There were no significant effects of time $\left(\mathrm{F}_{(1,176,22,339)}=2,983\right.$, $\left.\mathrm{p}>0.05, \mathrm{n}_{\mathrm{p}}^{2}=0.136\right)$; however, there was a significant effect of the condition $\left(\mathrm{F}_{(2,38)}=144.029, \mathrm{p}<0.001\right.$, $\left.\mathrm{n}_{\mathrm{p}}^{2}=0.883\right)$. There was no significant interaction between time and condition $\left(\mathrm{F}_{(2,600,49,406)}=1.411, \mathrm{p}>0.05\right.$, $\left.\mathrm{n}_{\mathrm{p}}^{2}=0.069\right)$. This finding demonstrates that the treadmill speed was different under the conditions at all times $(\mathrm{p}<0.01)$ (Figure 2 ) but was maintained stably over time under each condition.

\section{Feeling scale}

There was a significant difference between verbal commands with respect to FS $\left(\mathrm{F}_{(1.486,28.238)}=19.843\right.$, $\left.\mathrm{p}=0.000, \mathrm{n}_{\mathrm{p}}^{2}=0.511\right)$. FS was significantly higher in LI $\mathrm{x}$ HI $(\mathrm{p}<0.01, \mathrm{~d}=0.977)$ and MI x HI $(\mathrm{p}<0.01$, $\mathrm{d}=0.637)$; however, there were no differences between LI $x$ MI $(p>0.05, d=0.38)$. There were significant effects of time, $\left(\mathrm{F}_{(2,352,44,689)}=7.065, \mathrm{p}<0.01, \mathrm{n}_{\mathrm{p}}^{2}=0.271\right)$, and

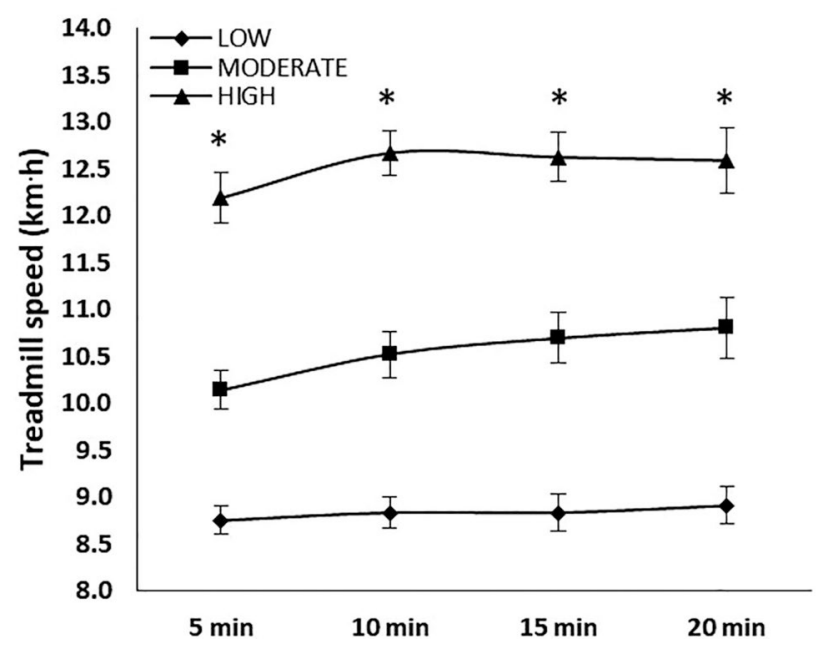

Figure 2 - Treadmill speed during low, moderate, and high verbal commands. * Significant difference under all conditions. 
condition, $\left(\mathrm{F}_{(2,38)}=13.738, \mathrm{p}<0.001, \mathrm{n}_{\mathrm{p}}^{2}=0.420\right)$. In addition, there was a significant interaction between time and condition, $\left(\mathrm{F}_{(5,035,95,672)}=9.041, \mathrm{p}<0.001\right.$, $\mathrm{n}_{\mathrm{p}}^{2}=0.322$ ). This finding demonstrates that the FS was different at the condition at time 5 and $10 \mathrm{~min}$ (HI x LI), and 15 and $20 \min (\mathrm{HI} \times \mathrm{MI}, \mathrm{LI})(\mathrm{p}<0.01)$ (Figure 3). The effect of the time was observed under the conditions $\mathrm{MI}$ and HI. The FS were higher in post $5 \mathrm{~min}$ and post 10 min from baseline $(\mathrm{p}<0.01)$ to MI, and lower at time 15 and 20 min from post 5 and post $10(\mathrm{p}<0.01)$ to HI.

\section{Felt arousal scale}

There were differences between verbal commands in FAS $\left(\mathrm{F}_{(2,38)}=4.788, \mathrm{p}=0.017, \mathrm{n}_{\mathrm{p}}^{2}=0.201\right)$. FAS was significantly higher in $\mathrm{HI} x \mathrm{MI}(\mathrm{p}<0.05, \mathrm{~d}=0.5)$ and $\mathrm{HI} \mathrm{x}$ LI $(p<0.05, d=0.5)$; however, there were no differences between LI x MI $(p>0.05, d=0)$. There were significant effects of time $\left(\mathrm{F}_{(2,154,40,927)}=15.145, \mathrm{p}<0.01\right.$, $\left.\mathrm{n}_{\mathrm{p}}^{2}=0.444\right)$ and condition $\left(\mathrm{F}_{(2,38)}=4.666, \mathrm{p}<0.05\right.$, $\left.\mathrm{n}_{\mathrm{p}}^{2}=0.197\right)$. There was no significant interaction between time and condition, $\left(\mathrm{F}_{(5,098,96,864)}=1.990, \mathrm{p}>0.05\right.$, $\left.\mathrm{n}_{\mathrm{p}}^{2}=0.095\right)$. This finding demonstrates that the FAS was different at the condition at time $5 \mathrm{~min}(\mathrm{HI} \mathrm{x} \mathrm{MI}), 10 \mathrm{~min}$ (HI x MI, LI), and post $5 \mathrm{~min}(\mathrm{HI} \times \mathrm{LI})(\mathrm{p}<0.01)$ (Figure 4). The effect of time was observed under all conditions. The FAS scores were different at $20 \mathrm{~min}$ from baseline; at 15 and $20 \mathrm{~min}$ from that post $5 \mathrm{~min}$; and 10 , 15 , and $20 \mathrm{~min}$ from post $10 \mathrm{~min}(\mathrm{p}<0.01)$ to LI. In MI, there were differences at $5,10,15$, and 20 min from baseline $(\mathrm{p}<0.01)$. In HI, there were differences at $5,10,15$, and $20 \mathrm{~min}$ from baseline $(\mathrm{p}<0.01)$; at 10 and $20 \mathrm{~min}$ from post 5; and at 5, 10, 15, and $20 \mathrm{~min}$ from post 10 $(\mathrm{p}<0.01)$.

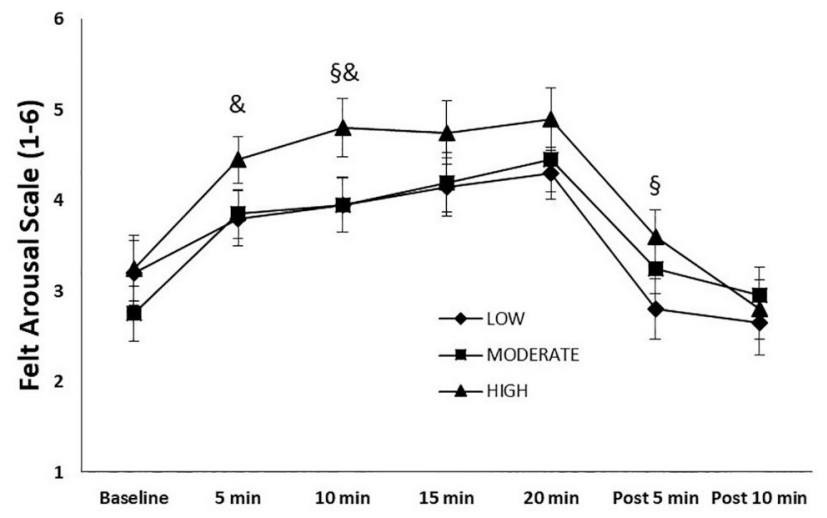

Figure 4 - Felt Arousal Scale during low, moderate, and high verbal commands. § Significant difference between High and Low. \& Significant difference between High and Moderate.

\section{Circumplex model}

In all verbal commands, the participants began with a sense of calmness (Figure 5) and after 5 min were in an energy sense, which remained throughout the exercise session. Post $5 \mathrm{~min}$, all returned to a sense of calmness. The verbal command high approached the tiredness quadrant toward the end of the exercise.

\section{Discussion}

The present study aimed to compare the effects of different verbal commands on the perceptual, affective, and physiological responses during running. The results demonstrated that perceptual responses and physiological were different for all verbal commands, with the intensity increasing as per the verbal commands given for running. The affective responses were positive during all verbal commands; however, they were lower in high verbal commands than in low and moderate verbal commands.

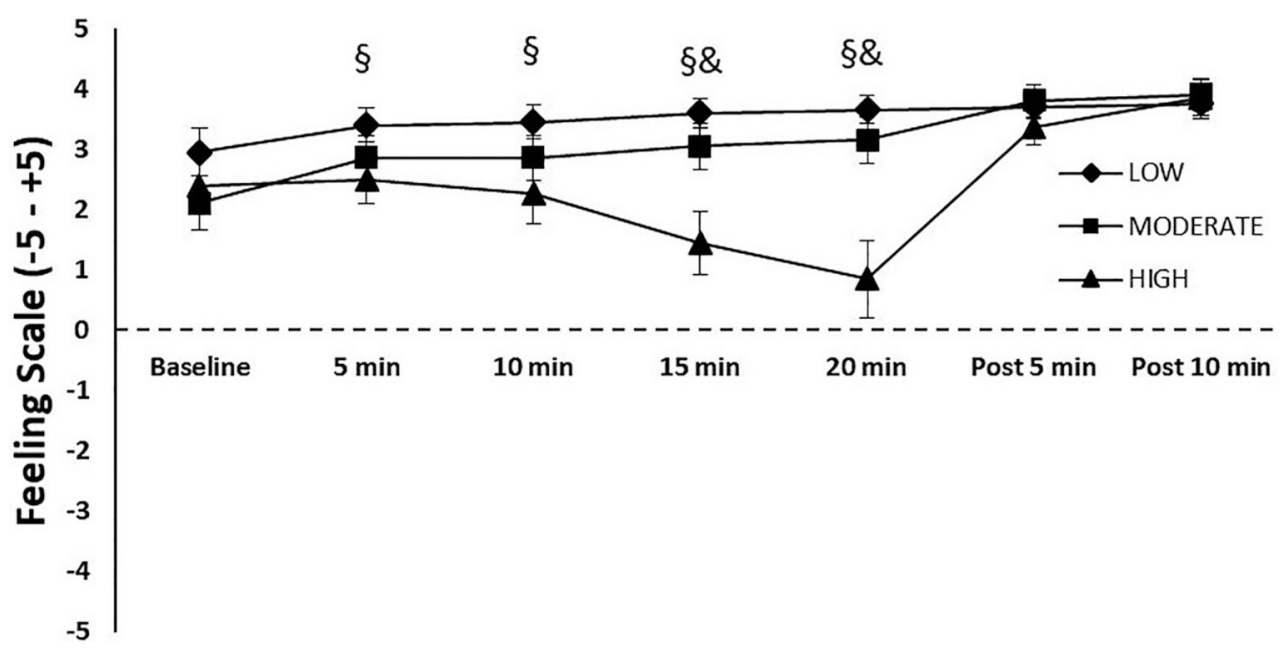

Figure 3 - Feeling Scale during low, moderate, and high verbal commands. § Significant difference between High and Low. \& Significant difference between High and Moderate. 

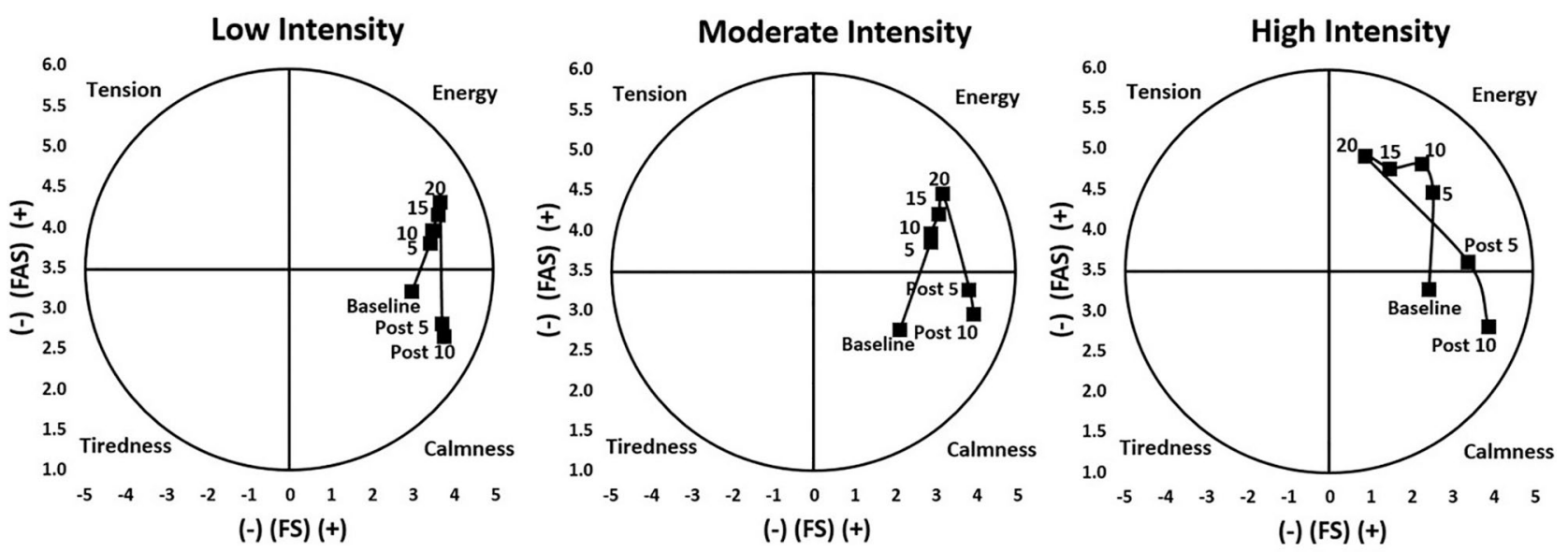

Figure 5 - Circumplex model to affective responses during low, moderate, and high verbal commands.

The perceptual and physiological parameters showed that the participants were able to adjust the running intensity in each intensity zone, proposed by the verbal commands, resulting in different perceptual and cardiovascular stimuli, in close agreement with the results reported by Kilpatrick, Robertson ${ }^{26}$. Our findings are consistent with the exercise recommendations proposed by Garber, Blissmer ${ }^{19}$ to improve health parameters and physical fitness, falling within the zones of moderate and vigorous-intensity. Even the lowest (low) command intensity used in the study provided cardiovascular stimulus within the intensity range considered moderate recommended by Garber, Blissmer ${ }^{19}$, demonstrating that the low, moderate, and high verbal commands can be used in the exercise prescription to improve cardiorespiratory fitness.

In studies that used self-selected intensity as an exercise prescription strategy, Lind, Joens-Matre ${ }^{18}$ observed that inactive women choose a $\%$ speed $(70 \%$ of peak speed) and $\% \mathrm{HR}\left(78.5 \% \mathrm{HR}_{\text {peak }}\right)$ similar to those in the present study for moderate $\left(68.8 \%\right.$ treadmill speed $\left._{\text {max }}\right)$ and low $\left(76.5 \% \mathrm{HR}_{\max }\right)$ verbal commands. Dias, Simao ${ }^{22}$, observed results for speed and $\% \mathrm{HR}_{\max }$ corresponding to the moderate verbal command $\left(10.5 \mathrm{~km} \cdot \mathrm{h}^{-1}\right.$ and $83.1 \%$ $\left.\mathrm{HR}_{\max }\right)$. Rose and Parfitt ${ }^{21}$ found a \% HR below the low command for inactive women $\left(69.8 \% \mathrm{HR}_{\max }\right)$, and values corresponding to the moderate command in active women $\left(81 \% \mathrm{HR}_{\max }\right)$. Previous studies have shown that inactive and active people tended to self-select intensities corresponding to low and moderate verbal command zones, respectively, ranges which promoted the most favorable affective responses.

The affective responses were positive throughout the exercise sessions prescribed by verbal commands; however, the high verbal command showed significant differences in relation to the low and moderate commands. These results are consistent with the Dual Mode Theory, indicating that affective responses are determined by the interaction between cognitive and interoceptive para- meters ${ }^{12,27}$. The importance of these parameters changes according to the intensity of the exercise. At intensities below or close to the VT, cognitive parameters are dominant, and affective responses tend to be positive. At the VT or intensities slightly above it, the affective responses can present great inter-individual variability, with the sensation of pleasure increasing in some individuals and decreasing in others. Above the VT, interoceptive parameters predominate, and most individuals experience a decreased sensation of pleasure ${ }^{12,17,27}$. In an investigation by Freitas, Ferreira ${ }^{7}$ that compared the affective responses during 12 weeks in self-selected and imposed intensity exercise ( $10 \%$ above the ventilatory threshold) in obese women, their results demonstrated that the group that performed the exercise sessions $10 \%$ above the ventilatory threshold showed lower affective responses, reaching negative values throughout the training period.

In addition to the postulates of the Dual Model Theory, studies have shown that from the self-selected intensity, there may still be an increase in exercise intensity, with consequent maintenance or reduction of affective responses, without reaching negative values. When comparing the affective responses of the self-selected exercise, and at an intensity, $10 \%$ above the values obtained in the self-selection, Ekkekakis and Lind $^{20}$ observed that in overweight women, exercise prescribed at $10 \%$ above the self-selected intensity decreased affective responses (without reaching negative values), whereas, in women with normal weight, affective responses did not change. Lind, Ekkekakis ${ }^{28}$ found in sedentary women that prescribed exercise $10 \%$ above the self-selected intensity $\left(91 \% \times 84 \% \mathrm{HR}_{\max }\right)$ decreased affective responses, without reaching negative values. In the present study, while the moderate verbal command presented intensities like some studies that used self-selected intensity, the high verbal command presented an intensity about $10 \%$ above the moderate verbal command $\left(90.6 \%\right.$ vs $\left.83.1 \% \mathrm{HR}_{\max }\right)$. These Findings showed a decline in the affective respon- 
ses of the high verbal command, without reaching negative values, demonstrating that this zone of intensity reached values around the VT, allowing for greater stimulation of cardiorespiratory fitness, but within an acceptable limit that permitted affective responses to remain positive.

The affective responses shown by the circumplex model demonstrate that the changes in the affective quadrants occurred similarly in all sessions. From baseline in the calm quadrant at the beginning of exercise, the FAS increased, remaining in the energy quadrant throughout the exercise session and returning immediately to the calm quadrant after the end of the session. Although the FS decreased from $10 \mathrm{~min}$ of exercise until the end of the exercise, this reduction was insufficient to reach the tension quadrant. These results demonstrate that $20 \mathrm{~min}$ of exercise prescribed by the low, moderate, and high verbal commands were adequate to increase the FAS to the energy quadrant and maintain the FS in positive values. Negative responses to FS could be a negative experience and induce exercise dropout. In contrast, high arousal may indicate attention; this is not necessarily a negative result $^{29}$. The permanency of the affective responses in the energy quadrant indicates that the exercise is enjoyable; however, the exercise performed in the tension quadrant could represent an unpleasant and negative experience.

The present study has certain limitations that must be considered when generalizing the results. The sample comprised of active men, aged 18-50 years, who regularly practiced aerobic exercise and running activities. The effect of the large age range was minimized by recruiting participants with similar physical fitness and exercise routines. The results may not be the same for women, overweight people, inactive people, or practitioners of other exercise modalities. In addition, the prescription of the verbal command above $20 \mathrm{~min}$ of exercise may present differences in affective responses, especially with the high verbal command ${ }^{30}$. Future investigations should explore the potential of exercise prescribed by verbal commands for different populations, exercise modalities, and exercise duration, in order to obtain relevant information about the scope of this exercise prescription methodology.

\section{Conclusion}

The results of the present study suggest that verbal commands can be used in running prescriptions. The verbal commands low/moderate/high can provide the cardiorespiratory stimulus of moderate or vigorous intensity without promoting negative affective responses. The verbal command "Moderate Intensity" can promote greater perceptual and physiological stimuli than "Low Intensity," without causing significant changes in affective responses.

\section{Acknowledgments}

The manuscript received financial support from Instituto Federal do Espírito Santo (IFES).

\section{References}

1. Haile L, Gallagher M, Robertson RJ. Perceived exertion laboratory manual: from standard practice to contemporary application, 2015.

2. Noble BJ, Robertson RJ. Perceived Exertion. Champaign: Human Kinetics Books; 1996.

3. Robertson RJ, Noble BJ. Perception of physical exertion: methods, mediators, and applications. Exerc Sport Sci Rev. 1997;25:407-52.

4. Morgan WP. Utility of Exertional Perception with Special Reference to Underwater Exercise: International J Sport Psychol. 2001;32: 137-61.

5. Lind E, Welch AS, Ekkekakis P. Do 'mind over muscle' strategies work? Examining the effects of attentional association and dissociation on exertional, affective, and physiological responses to exercise. Sports Med. 2009;39(9):74364.

6. Almeida FA, Nunes RF, Ferreira SS, Krinski K, Elsangedy $\mathrm{HM}$, Buzzachera CF, et al. Effects of musical tempo on physiological, affective, and perceptual variables and performance of self-selected walking pace. J Phys Ther Sci. 2015;27(6):1709-12.

7. Freitas LA, Ferreira SS, Freitas RQ, Souza CH, Garcia ED, Silva SG. Effect of a 12-week aerobic training program on perceptual and affective responses in obese women. J Phys Ther Sci. 2015;27(7):2221-4.

8. Halperin I, Emanuel A. Rating of Perceived Effort: Methodological Concerns and Future Directions. Sports Med. 2020;50(4):679-87.

9. Reed JL, Pipe AL. Practical Approaches to Prescribing Physical Activity and Monitoring Exercise Intensity. Can J Cardiol. 2016;32(4):514-22.

10. Ekkekakis P, Parfitt G, Petruzzello SJ. The pleasure and displeasure people feel when they exercise at different intensities: decennial update and progress towards a tripartite rationale for exercise intensity prescription. Sports Med. 2011;41(8):641-71.

11. Parfitt G, Hughes S. The Exercise Intensity-Affect Relationship: Evidence and Implications for Exercise Behavior. J Exerc Sci Fitness. 2009;7(2):34-41.

12. Ekkekakis P. Pleasure and displeasure from the body: Perspectives from exercise. Cogn Emot. 2003;17(2):213-39.

13. Sheppard KE, Parfitt G. Acute affective responses to prescribed and self-selected exercise intensities in young adolescent boys and girls. Pediatr Exerc Sci. 2008;20(2):12941.

14. Rose EA, Parfitt G. Pleasant for some and unpleasant for others: a protocol analysis of the cognitive factors that influence affective responses to exercise. Int J Behav Nutr Phys Act. 2010;7:15.

15. Vandoni M, Codrons E, Marin L, Correale L, Bigliassi M, Buzzachera CF. Psychophysiological Responses to Group Exercise Training Sessions: Does Exercise Intensity Matter? PLoS One. 2016;11(8):e0149997. 
16. Timme S, Brand R. Affect and exertion during incremental physical exercise: Examining changes using automated facial action analysis and experiential self-report. PLoS One. 2020;15(2):e0228739.

17. Ekkekakis P. Let them roam free? Physiological and psychological evidence for the potential of self-selected exercise intensity in public health. Sports Med. 2009;39 (10):857-88.

18. Lind E, Joens-Matre RR, Ekkekakis P. What intensity of physical activity do previously sedentary middle-aged women select? Evidence of a coherent pattern from physiological, perceptual, and affective markers. Prev Med. 2005;40(4):407-19.

19. Garber CE, Blissmer B, Deschenes MR, Franklin BA, Lamonte MJ, Lee IM, et al. American College of Sports Medicine position stand. Quantity and quality of exercise for developing and maintaining cardiorespiratory, musculoskeletal, and neuromotor fitness in apparently healthy adults: guidance for prescribing exercise. Med Sci Sports Exerc. 2011;43(7):1334-59.

20. Ekkekakis P, Lind E. Exercise does not feel the same when you are overweight: the impact of self-selected and imposed intensity on affect and exertion. Int J Obes (Lond). 2006;30 (4):652-60.

21. Rose EA, Parfitt G. Exercise experience influences affective and motivational outcomes of prescribed and self-selected intensity exercise. Scand J Med Sci Sports. 2012;22(2):26577.

22. Dias MRC, Simao R, Saavedra FJF, Buzzachera CF, Fleck S. Self-Selected Training Load and RPE During Resistance and Aerobic Training Among Recreational Exercisers. Percept Mot Skills. 2018;125(4):769-87.

23. Hardy CJ, Rejeski WJ. Not what, but how one feels: The measurement of affect during exercise. J Sport Exerc Psychol. 1989;11(3):304-17.

24. Svebak S, Murgatroyd S. Metamotivational dominance: a multi-method validation of reversal theory constructs. J Pers Soc Psychol 1985;48:107-116.

25. Utter AC, Robertson RJ, Green JM, Suminski RR, McAnulty SR, Nieman DC. Validation of the adult OMNI Scale of perceived exertion for walking/running exercise. Med Sci Sports Exerc. 2004;36(10):1776-80.

26. Kilpatrick MW, Robertson RJ, Powers JM, Mears JL, Ferrer NF. Comparisons of RPE before, during, and after selfregulated aerobic exercise. Med Sci Sports Exerc. 2009;41 (3):682-7.

27. Ekkekakis P. The Dual-Mode Theory of affective responses to exercise in metatheoretical context: II. Bodiless heads, ethereal cognitive schemata, and other improbable dualistic creatures exercising. Int Rev Sport Exerc Psychol. 2009;2 (2):22.

28. Lind E, Ekkekakis P, Vazou S. The affective impact of exercise intensity that slightly exceeds the preferred level: 'pain' for no additional 'gain'. J Health Psychol. 2008;13(4):4648 .

29. Oliveira BR, Slama FA, Deslandes AC, Furtado ES, Santos TM. Continuous and high-intensity interval training: which promotes higher pleasure? PLoS One. 2013;8(11):e79965.

30. Martinez N, Kilpatrick MW, Salomon K, Jung ME, Little JP. Affective and enjoyment responses to high-intensity interval training in overweight-to-obese and insufficiently Active Adults. J Sport Exerc Psychol. 2015;37(2):138-49.

\section{Corresponding author}

Sandro dos Santos Ferreira. Rua Augusto Costa de Oliveira 660, 29285-000 Piúma, ES, Brazil.

E-mail: sandroferreiraef@hotmail.com; sandro.ferreira@ifes.edu.br.

Manuscript received on March 31, 2021

Manuscript accepted on July 22, 2021

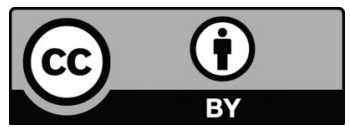

Motriz. The Journal of Physical Education. UNESP. Rio Claro, SP, Brazil - eISSN: 1980-6574 - under a license Creative Commons - Version 4.0 